\title{
Angewandte Landschaftsforschung
}

\section{Einleitung}

1977 bat das Eidgenössische Departement des Innern den Schweizerischen Schulrat, eine Gruppe für angewandte Landschaftsforschung zu schaffen. Diese wurde an der Eidgenössischen Anstalt für das forstliche Versuchswesen (EAFV) - eine der fünf mit den Eidgenössischen Technischen Hochschulen verbundenen Anstalten - in Birmensdorf bei Zürich eingerichtet. Zwischen dem Sommer 1977 und dem Frühjahr 1978 nahmen ein Zoologe, ein Geograph (der Autor) und ein Geobotaniker die Arbeit an der EAFV auf. Der Auftrag enthält die Zweckbestimmung, es seien Grundlagen für den Naturund Landschaftsschutz zu erarbeiten, und es sei koordinierend und fördernd im Bereich der angewandten Landschaftsforschung zu wirken.

Der beschränkte Raum erlaubt es nicht, Landschaftsforschung in den Rahmen der heute verfügbaren Literatur zu stellen. Deshalb kann hier nur ein grober Überblick skizziert werden.

\section{Begriffserläuterungen}

Um den Einstieg zu geben, ist kurz zu erläutern, wie angewandte Landschaftsforschung im folgenden zu verstehen ist. Landschaft wird als System interpretiert, das aus Elementen aufgebaut ist, welche miteinander in Beziehung stehen. Dabei kann es sich um Naturlandschaft oder um die viel häufigere Kulturlandschaft handeln. Größe, Lage u. ä. der zu untersuchenden Landschaft spielen keine Rolle.

Entscheidend für die Landschaftsforschung ist es, wie die Grenzen der Systeme, die bezüglich ihrer Elemente und Beziehungen eine relativ hohe räumliche und zeitliche Geschlossenheit aufweisen, festgelegt werden. Daß Auswirkungen menschlicher Tätigkeiten im landschaftlichen System ebenfalls erfaßt werden, ist hier nur deshalb anzumerken, weil es Arbeiten im Bereich Landschaft gibt, die den Menschen nahezu ausklammern.

Der Begriff "angewandt» bedeutet nichts anderes, als daß Ergebnisse oder Teilerkenntnisse von Untersuchungen gegebenenfalls bis zu einer anwendbaren Form auszuarbeiten sind. Angewandte For- schung als solche gibt es bei der geforderten ganzheitlichen Betrachtungsweise nicht; eine Untersuchung, von einer klaren Fragestellung oder von $\mathrm{Hy}$ pothesen ausgehend, kann grundsätzlich nicht darauf abzielen, mit ihren Ergebnissen zum voraus festgelegter, spezifischer Anwendung zu dienen. Es ist lediglich möglich, Fragestellung und Untersuchungsanordnung so anzusetzen, daß anwendbare Ergebnisse erwartet werden können. Auftrags- und Zweckforschung, auf gutachterischem Wege entstanden, haben in neuerer Zeit im Sektor Natur und Landschaft eine Kluft gebildet zwischen der sogenannten Grundlagenforschung und der angewandten Forschung. Dieser Hiatus ist zu schließen, indem bereits Fragestellungen und Untersuchungsabläufe auf wissenschaftliche Erkenntnisse abzustützen sind. Die Ergebnisse haben wissenschaftlicher Überprüfung standzuhalten. Hinterher ist festzustellen, ob anwendbare Erkenntnisse gewonnen worden sind oder nicht.

\section{Gegenstand}

Das landschaftliche System besteht aus einer Menge von Elementen, die miteinander in Beziehung stehen. Als Beispiel diene eine frühere, naturnahe Flußlandschaft des Mittellandes mit mäandrierendem Fluß, Altläufen, Altwassern, Ufervegetation, Amphibienbeständen, einer Fischotterpopulation, lichten Auenwäldern und andern Naturbereichen. In einer solchen Landschaft, Teillandschaft - oder je nach Standpunkt auch Landschaftsausschnitt bestehen sichtbare und unsichtbare Beziehungen zwischen den Elementen; so zwischen dem Grundwasserstrom und dem ihn speisenden Fluß; zwischen dem episodisch überschwemmenden Fluß und den durch Erosion und Akkumulation initiierten Sukzessionen; zwischen dem Altwasser und dem Auenwald, indem ersteres als Laichplatz, letzterer als Jagdgebiet für Amphibien dient usw. Im letzten Jahrhundert waren solche Verhältnisse noch vorhanden, zum mindesten abschnittweise oder

Dr. Klaus C. Ewald, Geograph SIA, Eidg. Anstalt für das forstliche Versuchswesen, 8903 Birmensdorf. 
doch mit einer kleineren Zahl von ursprünglichen, natürlichen Elementen.

Im Laufe der Zeit wurden die Naturbereiche durch Kulturbereiche ersetzt. Zum mindesten wurden sie räumlich oder in ihrer Substanz verändert. Es wurden Flüsse begradigt, befestigt und eingedämmt; Auenwälder wurden gerodet und urbarisiert; Altläufe und Altwasser aufgefüllt und in Kulturland überführt usw. Einige wenige Reste von Naturelementen sind noch verblieben. Hinzugekommen sind aber kulturelle und zivilisatorische Elemente, wie Siedlungen, Industrieanlagen, Straßen, Kiesgruben und ähnliches. Auch im neuentstandenen kulturlandschaftlichen System bestehen Beziehungen. Diejenigen der Naturlandschaft sind kaum mehr oder nur noch teilweise vorhanden, da Naturelemente aufgehoben oder eingeengt worden sind. Neue Beziehungen beginnen sich einzuspielen, so jene zwischen der intensiven Düngung des Kulturlandes im ehemaligen Auenwald und der dadurch bedingten Beeinflussung der Grundwasserqualität. Eine andere offensichtlich neue Beziehung besteht in der Absenkung des Grundwasserspiegels durch bauliche Eingriffe. Das Grundwasser soll als Beispiel dienen, um auf übergeordnete Elemente in der Landschaft hinzuweisen, da Veränderungen des Grundwasserspiegels oft vorgenommen wurden. Im stark meliorierten Mittelland beeinflußt der durch wasserbauliche Maßnahmen abgesenkte Wasserspiegel mehrere Elemente gleichzeitig - dominiert in diesem Sinne also zahlreiche andere Einflüsse.

Folgende Momente sind dem vorhergehenden zu entnehmen: Landschaft unterliegt einer eigenständigen und einer durch den Menschen geschaffenen Dynamik. Weil Landschaft als System einer Anzahl miteinander in Beziehung stehender Elemente aufzufassen ist, ist sie weder als Summe räumlicher benachbarter Teile noch von einem momentanen $\mathrm{Zu}$ stand ausgehend zu begreifen. Landschaft ist deshalb gleichzeitig in Raum und Zeit zu untersuchen.

Eine Vielzahl von baulichen und strukturellen Maßnahmen bewirkt den Wandel von der Naturzur Kulturlandschaft. Die Veränderungen, die Natur- durch Kulturelemente ersetzen, stellen als solche einen Gegenstand der Landschaftsforschung dar; denn sie setzen durch ihre Auswirkungen auf einzelne Elemente und deren Beziehungen Veränderungen in Gang und werfen damit neue Probleme auf.

\section{Ziele}

Die Ziele der Landschaftsforschung bestehen im Festhalten und Darstellen von Veränderungen in prädiktivem Sinne. Dabei geht es einerseits darum, die Änderungen der Mengen der Elemente, d.h. die
Zustandsänderungen zu erfassen. Andererseits besteht ein Ziel im Auffinden der Änderung der Beziehungen zwischen den Elementen. Ausgehend von einer Reihe von Zustands- und Beziehungsänderungen ist mit einem abschätzbaren $\mathrm{Ma} ß$ an $\mathrm{Zu}$ verlässigkeit auf einen neuen Zustand zu schließen. Wesentlich dabei ist, Veränderungen derart unter Kontrolle zu bringen, daß Abläufe im landschaftlichen Geschehen erkennbar und gegebenenfalls voraussehbar werden.

\section{Aufgaben}

Die Veränderungen der Landschaft stellen also Gegenstand und Ziele der Landschaftsforschung dar. Zusammengefaßt bestehen die Aufgaben im Erfassen von Zuständen zu verschiedenen Zeitpunkten in verschiedenen Räumen. Diese Aufgaben seien anhand einiger Ansätze skizziert.

\subsection{Veränderung der sichtbaren Elemente der Landschaft}

Am einfachsten läßt sich dies am Beispiel eines Grundrisses der Landeskarte der Schweiz 1:25000 erläutern. Ein beliebiger Kartenausschnitt auf einer Erstausgabe dieses Kartenwerkes ist mit demselben Ausschnitt auf der neuesten Ausgabe zu vergleichen. Die dazwischen eingetretenen Veränderungen lassen sich feststellen: Straßen und Häuser sind gebaut worden; der Streuobstbau ist aufgegeben oder durch Obstintensivanlagen ersetzt worden usw. Markiert man sämtliche Veränderungen gegenüber der Erstausgabe in der neuesten Ausgabe, so kann man diese quantifizieren, so z. B. die Länge der neu gebauten Straßen, die Fläche der überbauten Areale, die Länge der eingedolten Fließgewässer, die Fläche der entwässerten Feuchtgebiete usw. Das ist eine, zwar maßstäblich begrenzte Möglichkeit, Veränderungen in Raum und Zeit festzuhalten. Mit dieser Art des Vergleichens und Quantifizierens ist aber nur die Veränderung der sichtbaren Elemente, nicht aber die Änderung der Beziehungen erfaßbar. Beim Kartenvergleich können nur Elemente, die Gegenstand der Kartographie sind, berücksichtigt werden. Verwendet man dagegen Luftbilder aus verschiedenen Zeiten, so sind auch qualitative Momente wie z. B. die Ausbildung der Vegetation in die Veränderungsdarstellung einzubeziehen. Auch diese Veränderungen lassen sich quantifizieren. Man kann sie - wie die erstgenannten - bewerten, indem man sie z. B. auf eine Fläche und einen Zeitraum bezieht.

\subsection{Auswirkungen der Veränderungen der sichtbaren Elemente der Landschaft}

Der Veränderung der sichtbaren Elemente folgen Änderungen der Beziehungen, die schwer voraus- 
sehbar sind - deren Folgen hingegen sichtbar werden können. Zustandsänderungen der Landschaft lassen sich qualitativ mit Stich-oder Schlagworten ausdrücken, wobei diese sowohl eine Veränderung von sichtbaren Elementen, als auch daraus resultierende Beziehungsänderungen signalisieren. Begriffe wie Begradigung, Eindämmung, Eindolung, Entwässerung, Intensivierung usw. bringen die eingeleiteten Prozesse, die eingetretene Veränderung sowie die Änderung der Beziehungen zum Ausdruck.

Beziehungsänderungen werden meist erst realisiert, wenn dadurch neue Beziehungen entstanden sind. Am einleuchtendsten sind Beispiele im Bereich der «Schädlinge». So sind im Zuge der Landschaftsveränderungen natürliche Futterplätze mit Samen, Früchten, Beeren usw. beseitigt worden; als Folge davon weichen gewisse Vogelarten auf Kulturpflanzen aus. Der Rückgang des Wiedehopfs scheint eine Folge der Beseitigung der parkartigen Obstanlagen, der Entwässerung und der Weideintensivierung zu sein. Durch die Intensivierung der Graswirtschaft sind blumenreiche Wiesen und die auf sie angewiesenen Kleintiere seltener geworden. Diese wenigen aus der Vielzahl heute bekannter Beispiele sollen genügen, um die altbekannte, aber nicht zur Kenntnis genommene Tatsache zu wiederholen: ein einzelnes Element ist nicht isoliert manipulierbar. Eindrücklich zeigt sich die Verkettung von Beziehungen bei der Änderung der Menge des Elementes Wasser in einem Hochmoor: Nährstoffhaushalt, Hochmoorpflanzen, Torfbildung und damit das Relief werden beeinflußt. Analoges ereignet sich bei der Eutrophierung eines Sees. Die Ursache für dessen Störung liegt häufig in einer zu hohen Phosphateinschwemmung. Dies führt zu stärkerem Algenwachstum und damit zu größerem Sauerstoffverzehr. Parallel dazu nimmt die Sichttiefe ab, die Kleinkrebse und die Fischmenge (über die Veränderung der Artenzusammensetzung ist damit nichts ausgesagt) nehmen zu.

\subsection{Ursachen für Störungen}

Die Suche nach Beziehungen erfolgt über das Bestimmen von Änderungsraten und Korrelationen. Sie ist zunächst häufig unter biologischen Gesichtspunkten anzugehen. Doch zeigt die Erfahrung über die Umweltveränderungen der letzten Jahre, daß auslösende Momente im Landschaftswandel zu finden sind. Immer wieder "ereignen» sich unkontrollierte, unvorhergesehene Änderungen, weil das an technische Abläufe gewohnte Vorstellungsvermögen die Folgen von mehreren veränderten Faktoren und Elementen nicht verarbeiten und voraussehen kann. Es ist daher im Rahmen der Landschaftsforschung nach Ursachen für Störungen (meist Gleichgewichtsstörungen) zu suchen.
Ausgangslage für die Untersuchung heutiger Verhältnisse können schweizerische mittelländische Landschaften der fünfziger Jahre darstellen. Damals - z.T. noch zu Beginn der sechziger Jahre waren diese landschaftlichen Systeme von verschiedenen Naturbereichen durchsetzt. Zum einen war die landwirtschaftliche Nutzung bezüglich Mechanisierung und Hilfsstoffeeinsatz noch nicht intensiv; zum andern gab es extensiv genutzte Bereiche und kaum genutztes «Niemandsland» am Rande von Acker- und Wiesengebieten, den Waldrändern entlang, an Straßenböschungen usw. Die bewirtschafteten Gebiete uferten in Naturbereiche aus; zusätzlich gab es wesentlich mehr Reste der Naturlandschaft, wie unkorrigierte Gewässer, Auenbereiche, Feuchtgebiete usw.

Weder die räumliche Ausnutzung noch die Nutzung der einzelnen Flächen waren intensiv. Außerdem gab es erhebliche Unterschiede in der Nutzungsintensität. Deshalb waren in großen Teilen der Landschaft eigentliche Naturbereiche und natürliche Bereiche vorhanden. Daß zwischen Naturraum und Kulturraum Beziehungen spielen konnten, war kein Zufall, lehnten sich frühere Nutzungsbereiche viel inniger an natürliche Gegebenheiten an als die heutigen. So konnten Kulturlandschaften ein eigentliches Mosaik an Lebensräumen enthalten.

Mit diesen Landschaftszuständen sind die im Abschnitt 5.1 umrissenen Veränderungen zu konfrontieren. Die in Abschnitt 5.2 eingeführten Schlagworte kennzeichnen die Entwicklung: Bauten aller Art, Korrektionen, Begradigungen, Eindämmungen, Rationalisierung, Intensivierung und ähnliches sind erstellt oder durchgeführt worden. Parallel dazu gewann man die Einsicht, Raumordnung sei durch Raumplanung zu erreichen. Deshalb versucht man, die besiedelten und zu besiedelnden Gebiete wie auch die nicht überbaute Landschaft in den planerischen Griff zu bekommen. Dabei geht es darum, Nutzungsbereiche zu erobern, zu verteidigen und gegen andere Nutzungsbereiche abzugrenzen. Nutzung der Landschaft bedeutet nichts anderes als bestimmte Ansprüche wahrzunehmen. Deshalb sind Nutzungsbereiche als Interessenssphären in der Landschaft zu verstehen. Das führt zur Einteilung der Landschaft in Nutzungsbereiche wie Wald, Landwirtschaftsgebiet, Wohnzonen, Industriezonen usw. Der Detaillierungsgrad ist dort am größten, wo sich die Interessen am meisten konkurrenzieren, nämlich in den Baugebieten. Außerhalb der Baugebiete wird oft nur eine Zone, das sogenannte übrige Gemeindegebiet, ausgeschieden. Es kommt aber trotzdem hie und da eine Differenzierung vor, sei das im Rahmen einer Zonen- oder gar einer Landschaftsplanung.

Aus der Sicht der Landschaftsforschung ist das Rechtsinstrument der Planung und Verwaltung der 
Landschaft irrelevant. Wesentlich ist, ob bei der Beplanung der Landschaft den Lebensräumen, den natürlichen Gegebenheiten, den Beziehungen usw. Rechnung getragen wird oder nicht. Der Alltag beweist, daß beim Aufteilen der Landschaft in Nutzungsbereiche nur die materielle Werthierarchie zum Tragen kommt, indem Landschaft den Nutzungen angepaßt wird. Namentlich strukturbereinigte und meliorierte Landschaft belegt, daß mit dieser Aufteilung der Landschaft und dem Statuieren von scharfen Grenzen, anstelle von Übergängen, Instabilitäten und Unstetigkeiten (begradigte Waldränder, steile Böschungen, rasche Wasserabzüge usw.) geschaffen werden. Den Umstrukturierungen der Landschaft, die sich mit Blockwürfen, Drainagen, Planierungen usw. manifestieren und von Intensivierungen aller Art gefolgt werden, fallen Lebensräume, Elemente und Beziehungen zu Hauf zum Opfer. Anstelle der vormaligen Säume, der gleitenden Übergänge und des Niemandslandes sind Nutzungsbereiche mit scharfen Grenzen getreten. Dadurch werden vermehrt Nutzungsüberlagerungen in der Landschaft herbeigeführt. Diese können sich dann besonders negativ auswirken, wenn sie sich nicht an gegebene Naturräume wie Geländekammern, Flußläufe, Grundwasserkörper u.ä. halten.

Die Quintessenz ist folgendermaßen zu formulieren: Lebensräume stellen Elemente der Landschaft dar. Die Landschaftsforschung befaßt sich mit dem System dieser Lebensräume. Die menschlichen Nutzungsbereiche decken sich aber nur zum Teil mit ihnen. Solange sich planerische Maßnahmen nur mit dem Zuordnen von Nutzungsbereichen befassen und die Lebensräume unberücksichtigt lassen, solange wird es Gleichgewichtsstörungen und Konflikte geben. Ein Aufgabenbereich der Landschaftsforschung besteht deshalb darin, frühere, stabile Zustände einer Landschaft sowie die eingetretenen Veränderungen zu untersuchen, um Ursachen der Störung nachvollziehbar darstellen zu können.

\section{Grundlagen}

Es gibt verschwindend wenige Quellen, die landschaftliche Verhältnisse, Lebensräume und Lebensbedingungen früherer Zeitpunkte dokumentieren. Außerdem fehlt nachvollziehbares Grundlagenmaterial aus dem Bereich Landschaft weitgehend. Warum gebricht es der Landschaftsforschung an Grundlagen? Das liegt darin begründet, daß nur Tatbestände erfaßt werden, die unmittelbaren Nutzen bringen oder die in der Wertvorstellung als selbstverständlich figurieren. Statistische Jahrbücher und andere Zusammenstellungen dokumentieren das eindrücklich: Angaben über den Viehbe- stand, Erntestatistiken, das Verzeichnis der Kunstbauten der Nationalstraßen usw. werden sorgfältig nachgeführt. Wo aber kein unmittelbarer Nutzen besteht, gibt es wenig Nachfrage nach Wissen und somit keine finanzielle Unterstützung. So ist unbekannt, wieviele Hochmoore oder Davallseggenrieder heute in der Schweiz vorhanden sind; welches ganz allgemein die früheren und heutigen Verbreitungsgebiete von Pflanzen- und Tierarten sowie von Lebensgemeinschaften und Kleinlebensräumen sind. Das Wissen über Landschafts- und Naturzustände fehlt nicht nur für früher, sondern mit wenigen Ausnahmen auch für heute - die Schweiz ist in mancher Beziehung terra incognita. Deshalb steht die Landschaftsforschung vor unüberbrückbaren Wissenslücken; denn was nicht erfaßt wurde, bleibt unbekannt und ist als verloren zu qualifizieren.

\section{Arbeitsmethoden}

Da in der Landschaft physische, biologische und künstliche Elemente samt den Beziehungen untereinander bestehen, erfordert ihre Erforschung naturwissenschaftliche, kultur- und sozialgeographische Arbeitsmethoden, die einander möglichst breit überdecken müssen. So ist in der Landschaftsforschung ein inter- oder mindestens multidisziplinäres Arbeiten von Anfang an vonnöten. Das ist aber nur für Untersuchungen gegenwärtiger und zukünftiger Verhältnisse möglich. Veränderungen der ferneren Vergangenheit und der Landschaftsgeschichte können nur noch bruchstückweise derart nachgewiesen werden.

Kartenvergleiche und Luftbildauswertungen sind einfache Arbeitsmethoden, um Veränderungen sichtbarer Elemente zu erheben und um grobe Rekonstruktionsversuche landschaftlicher Zustände durchzuführen. Das einleuchtend erscheinende Vorgehen, Zahlenmaterial früherer Jahre räumlich - auf die Landschaft bezogen - auszuwerten, scheitert meistens daran, daß sich die Angaben auf politisch begrenzte Flächen beziehen und deshalb keine Differenzierungen nach Naturräumen innerhalb einer Gemeinde oder eines Bezirkes zulassen. Die Wiederholung von geobotanischen und faunistischen Bestandesaufnahmen ist nur dann möglich und sinnvoll, wenn die Örtlichkeiten im Gelände eindeutig auffindbar und die damaligen Aufnahmemethoden wieder angewendet werden können, was nach bisherigen Versuchen selten der Fall ist.

Das punktuelle und disziplinär getrennte Feststellen von Veränderungen hat dazu beigetragen, daß in den letzen Jahren in der Schweiz Inventare entstanden oder an die Hand genommen worden sind, und zwar auf verschiedenen Ebenen (Regionen, Kantone, Bund), in verschiedenen Sachbereichen 
und von verschiedenen Initiatoren. Inventare sind wichtige Instrumente der Landschaftsforschung. Wenn sie hier unter dem Abschnitt der Arbeitsmethoden erwähnt sind, so deshalb, weil sie bezüglich der landschaftlichen Systemelemente und der Nachvollziehbarkeit den langfristigen Zielen der Landschaftsforschung nur dann gerecht werden können, wenn sie der Dynamik der Landschaft in Raum und Zeit gerecht werden. Ein zusätzlicher methodischer Gesichtspunkt spielt eine entscheidende Rolle: Inventare müssen zeitlich und räumlich korrelierbar und ohne zeitlichen Verzug greifbar sein. Ihr Inhalt muß zu Stichhaltigkeitsbeweisen von Beziehungen und Beziehungsänderungen beigezogen werden können. Deshalb drängt sich auch das Zugänglichmachen von Inventaren durch Datenbanken arbeitsmethodisch auf.

\section{Ausblick}

Die Ausdehnung der Schweiz ist begrenzt; Menge und Dichte der Veränderungen scheinen aber unbegrenzt. Quantitatives leuchtet ein; Qualitatives wenig. Eine Philosophie und ihr entsprechende Leitbilder fehlen; wie soll die Landschaft in 20, 50 und 100 Jahren aussehen? Disneyland, vermarktete Nutzlandschaft oder Lebensraum? Verfassungsmä-
Bige und gesetzliche Instrumente sind vorhanden. Gesetzmäßigkeiten und Unwägbarkeiten könnten als Orientierungshilfen dienen. In der Werthierarchie müssen aber zuerst optimale, wünschenswerte Landschaftszustände als übergeordnete Zielsetzung Platz finden.

\section{Résumé}

La recherche appliquée en matière de paysage tente de cerner dans l'espace et le temps les sites naturels ou cultivés en tant que systèmes composés d'éléments étroitement liés. Les modifications du paysage font l'objet de la recherche car elles posent des problèmes nouveaux. C'est pourquoi son but est de prévoir ces changements: modifications dans le nombre des éléments ou dans l'état, ainsi que les changements de relations. L'application doit amener à prédire des situations nouvelles à partir d'une série de situations connues. De même, l'étude de l'état des sites dans le temps et l'espace fait partie des tâches de la recherche sur les sites, afin de déceler des changements de relations. Dans ce but on se sert de méthodes de travail issues des sciences naturelles et de la géographie humaine comprenant la comparaison de cartes, l'interprétation de photos aériennes et les inventaires sur le terrain. 